\title{
ON AN INFINITE-DIMENSIONAL VERSION OF THE KREISS MATRIX THEOREM
}

\author{
ANDRZEJ POKRZYWA \\ Institute of Mathematics, Polish Academy of Sciences \\ P.O. Box 137, Śniadeckich 8, 00-950 Warszawa, Poland \\ E-mail: AP@IMPAN.IMPAN.GOV.PL
}

The Kreiss matrix theorem with Spijker's improvement may be formulated as follows:

If $A$ is a linear operator acting in a normed d-dimensional space such that

$$
\left\|(A-\lambda)^{-1}\right\| \leq \frac{M}{|\lambda|-1} \quad \text { for }|\lambda|>1
$$

then

$$
\left\|A^{n}\right\| \leq e d M
$$

In this paper we are looking for some infinite-dimensional version of this theorem in Hilbert spaces. As a measure of "finite-dimensionality" we shall use crossnorms of compact operators.

The first lemma and its proof show difficulties in obtaining estimates of norms of powers of an operator.

Lemma 1. Suppose that $K$ is a compact operator such that (1) holds. Then $K$ is power bounded, i.e. $\sup _{n}\left\|K^{n}\right\|<\infty$.

Proof. Let $E$ be the eigenprojection of $K$ on the subspace spanned by all the generalized eigenvectors corresponding to the eigenvalues of modulus 1 . The Kreiss matrix theorem implies that

$$
\left\|\left.K^{n}\right|_{\operatorname{ran} E}\right\| \leq e M \operatorname{dim} \operatorname{ran} E .
$$

1991 Mathematics Subject Classification: 47D50, 47A10, 15A45.

The paper is in final form and no version of it will be published elsewhere. 
Since the spectral radius of $\left.K\right|_{\operatorname{ran}(1-E)}$ is equal to $\lim _{n \rightarrow \infty}\left\|\left.K^{n}\right|_{\operatorname{ran}(1-E)}\right\| \|^{1 / n}=$ $r<1$, there exists $M_{1}$ such that $\left\|\left.K^{n}\right|_{\operatorname{ran}(1-E)}\right\| \leq M_{1}$. Therefore

$$
\left\|K^{n}\right\|=\left\|\left.K^{n}\right|_{\operatorname{ran} E} E+\left.K^{n}\right|_{\operatorname{ran}(1-E)}(1-E)\right\| \leq e M \operatorname{dim} E\|E\|+M_{1}\|1-E\| .
$$

The dimension of the eigenspace ran $E$ may be effectively bounded for some classes of operators. A simple modification of the above proof shows also that

$$
\limsup _{n \rightarrow \infty}\left\|K^{n}\right\| \leq e M \operatorname{dim} E\|E\| .
$$

It follows from the proof that in order to estimate the norms of powers of $A$ it suffices to estimate the dimension and norm of the eigenprojection on the subspace spanned by eigenvectors corresponding to sufficiently large eigenvalues. This may be effectively done for operators with a bounded unitarily invariant crossnorm which is not equivalent to the operator norm. Let | | | be such a norm, defined on an ideal $\mathfrak{S}$.

Let $P_{n}$ be the orthogonal projection on an $n$-dimensional subspace. Since $\left|P_{n}\right| \rightarrow \infty$ as $n \rightarrow \infty$, the function $n(r)=\max \left\{n: r\left|P_{n}\right| \leq 1\right\}$ is a decreasing function of $r \in(0, \infty)$ and $n(r) \rightarrow \infty$ as $r \rightarrow 0$. Let $\mathcal{F}$ denote the set of finitedimensional operators. The adjoint crossnorm is defined by $|A|_{*}=\sup \{|\operatorname{tr} A X|$ : $X \in \mathcal{F},|X| \leq 1\}$. We set $\tau(r)=\mid P_{n(r)} \mathbf{|}_{*} \cdot \tau(r)$ is also a decreasing function.

It follows from [3] that

(i) there exists a function $\phi:[0, \infty) \times[0, \infty) \rightarrow[0, \infty)$ such that for any $A \in \mathfrak{S}$

$$
\left\|(A-\lambda)^{-1}\right\| \leq \phi(d, a) \leq \frac{3}{2 d} \exp \left(\frac{39}{d} a \tau\left(\frac{d}{6 a}\right)\right),
$$

where $d=d(\lambda, \sigma(A))=\inf _{\mu \in \sigma(A)}|\mu-\lambda|, a=|A|$. The function $\phi(d, a)$ is decreasing in $d$ and increasing in $a$,

(ii) if $\lambda_{1}, \lambda_{2}, \ldots$ are the eigenvalues of $A$ ordered in such a way that $\left|\lambda_{j}\right| \geq$ $\left|\lambda_{j+1}\right|$ and repeated according to multiplicity then

$$
\left|\lambda_{j}\right|<r|A| \text { for } j>n(r) .
$$

For the Schatten ideals $\mathfrak{S}_{p}(1 \leq p<\infty)$ the crossnorms $\|\cdot\|_{p}$ are defined by $\|A\|_{p}=\left(\sum_{j=1}^{\infty} s_{j}^{p}(A)\right)^{1 / p}$, where $s_{1}(A), s_{2}(A), \ldots$ are the singular values of $A$. For these crossnorms we can estimate the functions $n(r), \tau(r), \phi(d, a)$ as follows:

$$
\begin{gathered}
n_{p}(r) \leq r^{-p}, \\
\tau_{p}(r) \leq r^{p-1}, \\
\phi_{p}(d, a) \leq \frac{3}{2 d} \exp \left(\frac{39}{d} a\left(\frac{d}{6 a}\right)^{1-p}\right)=\frac{3}{2 d} \exp \left(39 \cdot 6^{p-1}\left(\frac{a}{d}\right)^{p}\right) .
\end{gathered}
$$

The theorem below is the main result of this paper.

THEOREM 1. Let \.| be a unitarily invariant crossnorm not equivalent to the operator norm, and $M \geq 1, a>0$. Then there exists $C>0$ such that for any 
$A \in \mathfrak{S}$ satisfying

$$
|A| \leq a \quad \text { and } \quad\left\|(A-\lambda)^{-1}\right\| \leq \frac{M}{|\lambda|-1} \quad \text { for }|\lambda|>1
$$

we have

$$
\left\|A^{n}\right\| \leq C .
$$

Pr o of. We assume that $a>1$; in the opposite case $\|A\| \leq|A| \leq 1$ and the assertion is trivial. Let $\lambda_{1}, \lambda_{2}, \ldots$ be the eigenvalues of $A$ arranged in decreasing order of their modulus. Let us fix $r \in(0,1)$; then for some $m \leq n(r / a)$ we have $1 \geq\left|\lambda_{1}\right| \geq\left|\lambda_{2}\right| \geq \ldots \geq\left|\lambda_{m}\right| \geq r>\left|\lambda_{m+1}\right|$. At least one of the numbers $1-\left|\lambda_{1}\right|$, $\left|\lambda_{1}\right|-\left|\lambda_{2}\right|, \ldots,\left|\lambda_{m-1}\right|-\left|\lambda_{m}\right|,\left|\lambda_{m}\right|-r$ is greater than $(1-r) /(m+1)$, therefore there exists $r_{1} \in(r, 1)$ such that the annulus ||$\lambda\left|-r_{1}\right|<(1-r) /(2(m+1))$ does not contain eigenvalues of $A$. Hence

$$
d(\lambda, \sigma(A)) \leq \frac{1-r}{2(m+1)} \quad \text { for }|\lambda|=r_{1} .
$$

This allows us to estimate the norm of the projection $E$ on the subspace spanned by the generalized eigenvectors corresponding to the eigenvalues with modulus greater than $r_{1}$. Namely, since

$$
I-E=\frac{-1}{2 \pi i} \int_{|\lambda|=r_{1}}(A-\lambda)^{-1} d \lambda,
$$

we have

$$
\|E\|=\|I-E\| \leq r_{1} \phi\left(\frac{1-r}{2(m+1)}, a\right) .
$$

Similarly we can estimate the norms of powers of $A_{1}=\left.A\right|_{\operatorname{ran}(1-E)}$. Since $\left|A_{1}\right| \leq$ |A| and $\sigma(A) \in K\left(0, r_{1}\right)$,

$$
\left\|A_{1}^{n}\right\|=\left\|\frac{-1}{2 \pi i} \int_{|\lambda|=1} \lambda^{n}\left(A_{1}-\lambda I\right)^{-1} d \lambda\right\| \leq \phi\left(\frac{1-r}{2(m+1)}, a\right) .
$$

The norms of powers of $A_{0}=\left.A\right|_{\mathrm{ran} E}$ may be estimated from the Kreiss matrix theorem: $\left\|A_{0}^{n}\right\| \leq e M \operatorname{dim} \operatorname{ran} E \leq e M m$.

These two inequalities give the desired estimate

$$
\begin{aligned}
\left\|A^{n}\right\| & =\left\|A_{0}^{n} E+A_{1}^{n}(I-E)\right\| \leq\left\|A_{0}^{n}\right\|\|E\|+\left\|A_{1}^{n}\right\|\|I-E\| \\
& \leq\left(\phi\left(\frac{1-r}{2(m+1)}, a\right)+e M m\right) \phi\left(\frac{1-r}{2(m+1)}, a\right)=C .
\end{aligned}
$$

For operators in Schatten ideals $\mathfrak{S}_{p}$ and $r=p /(1+p)$ we have $m \leq a^{p}((1+p) / p)^{p}$ $\leq e a^{p}, d=(1-r) /(2(m+1)) \geq 1 /\left(2 p\left(e a^{p}+1\right)\right), \phi(d, a) \leq 3\left(e a^{p}+1\right) \exp (39$. $\left.\overline{6}^{p-1}\left(2 p\left(e a^{p}+1\right) a\right)^{p}\right)$. Finally, $C=C_{p}=\left(\phi(d, a)+e^{2} M a^{p}\right) \bar{\phi}(d, a)$.

The obtained estimate has only theoretical significance; we shall obtain a better one for trace class operators. It is contained in the theorem below; instead 
of the operator norm we use its numerical radius $w(C)=\sup _{\|u\|=1}|\langle C u, u\rangle|$, which is equivalent to the norm: $\frac{1}{2}\|C\| \leq w(C) \leq\|C\|$. Inspecting Leveque and Trefethen's proof of the Kreiss matrix theorem one can find that it remains true with norms replaced by the numerical radius. The proof of the next theorem is given for a finite-dimensional Hilbert space - however, the estimate does not depend on dimension, and one can easily modify the proof to make it work in the infinite-dimensional case.

TheOREM 2. Suppose that $A$ is a trace class operator such that

then

$$
\|A\|_{1} \leq a, \quad w\left((A-\lambda I)^{-1}\right) \leq \frac{M}{|\lambda|-1}
$$

$$
w\left(A^{n}\right) \leq 2 a e M\left(4+\left(\frac{n+1}{n}\right) e^{8 a}\right) \quad \text { for } n=1,2,3, \ldots
$$

Proof. For a vector $u=\left(u_{1}, u_{2}, \ldots, u_{d}\right)^{\mathrm{T}}$, set $u^{*}=\left(\bar{u}_{1}, \bar{u}_{2}, \ldots, \bar{u}_{n}\right)$. Consider the determinant

$$
\operatorname{det}\left(\begin{array}{cc}
0 & u^{*} \\
u & B
\end{array}\right)
$$

with $B$ an invertible matrix and $u$ a unit vector. Without loss of generality we may assume that $u=(1,0, \ldots, 0)^{\mathrm{T}}$; then setting $P_{u}=I-\langle\cdot, u\rangle u, B_{u}=\left.P_{u} B\right|_{\operatorname{ran} P_{u}}$ we see that

$$
\operatorname{det}\left(\begin{array}{cc}
0 & u^{*} \\
u & B
\end{array}\right)=\operatorname{det}\left(\begin{array}{ccc}
0 & 1 & 0 \ldots 0 \\
1 & . & \ldots \\
0 & . & \\
\vdots & \vdots & B_{u} \\
0 & . &
\end{array}\right)=\operatorname{det}\left(B_{u}\right) .
$$

Note also that for any vector $v$,

$$
\operatorname{det} B_{u}=\operatorname{det}\left(\begin{array}{cc}
0 & u^{*} \\
u & B
\end{array}\right) \operatorname{det}\left(\begin{array}{cc}
1 & 0 \\
v & I
\end{array}\right)=\operatorname{det}\left(\begin{array}{cc}
u^{*} v & u^{*} \\
u+B v & B
\end{array}\right) .
$$

Setting here $v=-B^{-1} u$ we get

$$
\operatorname{det} B_{u}=\operatorname{det}\left(\begin{array}{cc}
-u^{*} B^{-1} u & u^{*} \\
0 & B
\end{array}\right)=-\left\langle B^{-1} u, u\right\rangle \operatorname{det} B .
$$

For $B=(A-\lambda I)^{-1}$ this identity implies that

$$
\left\langle(A-\lambda I)^{-1} u, u\right\rangle=-\frac{\operatorname{det}(A-\lambda I)_{u}}{\operatorname{det}(A-\lambda I)} .
$$

Let $\lambda_{i}, \lambda_{i}(u)$ denote the eigenvalues of the operators $A, A_{u}$ arranged in decreasing order of their modulus, respectively. We put $\lambda_{d}=0$, and then we can write

$$
\left\langle(A-\lambda)^{-1} u, u\right\rangle=-\frac{\prod_{j=1}^{d-1} \lambda_{j}(u)-\lambda}{\prod_{j=1}^{d} \lambda_{j}-\lambda}=\frac{1}{\lambda} \prod_{j=1}^{d} \frac{\lambda-\lambda_{j}(u)}{\lambda-\lambda_{j}} .
$$


Let $a=\|A\|_{1}$; then $\left\|A_{u}\right\|_{1} \leq a$, and it follows from (3) and (4) that for $m \leq a / r$,

$$
\left|\lambda_{j}\right| \leq r, \quad\left|\lambda_{j}(u)\right| \leq r \quad \text { for } j>m .
$$

We put

$$
\phi(\lambda)=\prod_{j \leq m} \frac{\lambda-\lambda_{j}(u)}{\lambda-\lambda_{j}}, \quad \psi(\lambda)=\prod_{j>m} \frac{\lambda-\lambda_{j}(u)}{\lambda-\lambda_{j}} .
$$

Note that if $|\lambda|=\varrho, r<\varrho$ then it follows from (5) that

$$
\begin{aligned}
|\psi(\lambda)| & =\prod_{j>m}\left|1+\frac{\lambda_{j}-\lambda_{j}(u)}{\lambda-\lambda_{j}}\right| \leq \prod_{j>m} \exp \left(\left|\frac{\lambda_{j}-\lambda_{j}(u)}{\lambda-\lambda_{j}}\right|\right) \\
& \leq \exp \left(\sum_{j>m} \frac{\left|\lambda_{j}\right|+\left|\lambda_{j}(u)\right|}{|\lambda|-\left|\lambda_{j}\right|}\right) \leq \exp \left(\frac{\sum_{j>m}\left|\lambda_{j}\right|+\left|\lambda_{j}(u)\right|}{\varrho-r}\right) \\
& \leq \exp \left(\frac{2 a}{\varrho-r}\right) .
\end{aligned}
$$

The same estimate may be obtained for $(\psi(\lambda))^{-1}=\prod_{j>m}\left(\lambda-\lambda_{j}\right) /\left(\lambda-\lambda_{j}(u)\right)$. Thus we have shown that

$$
\exp \left(\frac{-2 a}{\varrho-r}\right) \leq|\psi(\lambda)| \leq \exp \left(\frac{2 a}{\varrho-r}\right) \quad \text { for }|\lambda|=\varrho>r .
$$

Note further that

$$
\begin{aligned}
\psi^{\prime}(\lambda) & =\sum_{j>m}\left(\frac{\lambda-\lambda_{j}(u)}{\lambda-\lambda_{j}}\right)^{\prime}\left(\frac{\lambda-\lambda_{j}(u)}{\lambda-\lambda_{j}}\right)^{-1} \psi(\lambda) \\
& =-\psi(\lambda) \sum_{j>m} \frac{\lambda_{j}-\lambda_{j}(u)}{\left(\lambda-\lambda_{j}\right)\left(\lambda-\lambda_{j}(u)\right)},
\end{aligned}
$$

hence

(7)

$$
\begin{aligned}
\left|\psi^{\prime}(\lambda)\right| \leq|\psi(\lambda)| \sum_{j>m} \frac{\left|\lambda_{j}\right|+\left|\lambda_{j}(u)\right|}{\left(|\lambda|-\left|\lambda_{j}\right|\right)\left(|\lambda|-\left|\lambda_{j}(u)\right|\right)} \leq|\psi(\lambda)| \frac{2 a}{(\varrho-r)^{2}} & \\
& \text { for }|\lambda|=\varrho>r .
\end{aligned}
$$

Now we can estimate

$$
\left\langle A^{n} u, u\right\rangle=\frac{-1}{2 \pi i} \int_{\Gamma_{\varrho}} \lambda^{n}\left\langle(A-\lambda)^{-1} u, u\right\rangle d \lambda=\frac{-1}{2 \pi i} \int_{\Gamma_{\varrho}} \lambda^{n-1} \phi(\lambda) \psi(\lambda) d \lambda,
$$

where $\Gamma_{\varrho}$ is the circle with center 0 and radius $\varrho$.

Integrating by parts we get

$$
\left\langle A^{n} u, u\right\rangle=\frac{-1}{2 \pi i n} \int_{\Gamma_{\varrho}} \lambda^{n}\left(\phi^{\prime}(\lambda) \psi(\lambda)+\phi(\lambda) \psi^{\prime}(\lambda)\right) d \lambda .
$$


It follows from (7) that

$$
\left|\int_{\Gamma_{\varrho}} \lambda^{n} \phi(\lambda) \psi^{\prime}(\lambda) d \lambda\right| \leq \frac{4 \pi a}{(\varrho-r)^{2}} \varrho^{n+1} \max _{\lambda \in \Gamma_{\varrho}}\left|\phi(\lambda) \psi^{\prime}(\lambda)\right|,
$$

while by (6) and the Spijker Lemma in [4] we have

$$
\begin{aligned}
\left|\int_{\Gamma_{\varrho}} \lambda^{n} \phi^{\prime}(\lambda) \psi(\lambda) d \lambda\right| & \leq \varrho^{n} \exp \left(\frac{2 a}{\varrho-r}\right) \int_{\Gamma_{\varrho}}\left|\phi^{\prime}(\lambda)\right||d \lambda| \\
& \leq 2 \pi m \varrho^{n} \exp \left(\frac{2 a}{\varrho-r}\right) \max _{\lambda \in \Gamma_{\varrho}}|\phi(\lambda)|,
\end{aligned}
$$

and since by (6),

$$
|\phi(\lambda)| \leq|\phi(\lambda)| \cdot|\psi(\lambda)| \exp \left(\frac{2 a}{\varrho-r}\right)
$$

and $m \leq a / r$, we see that

$$
\left|\int_{\Gamma_{\varrho}} \lambda^{n} \phi^{\prime}(\lambda) \psi(\lambda) d \lambda\right| \leq \frac{2 \pi a \varrho^{n}}{r} \exp \left(\frac{4 a}{\varrho-r}\right) \max _{\lambda \in \Gamma_{\varrho}}|\phi(\lambda) \psi(\lambda)| .
$$

The above inequalities imply that

$$
\left|\left\langle A^{n} u, u\right\rangle\right| \leq \frac{1}{2 \pi n}\left(\frac{4 \pi a}{(\varrho-r)^{2}} \varrho^{n+1}+\frac{2 \pi a}{r} \exp \left(\frac{4 a}{\varrho-r}\right)\right) \max _{|\lambda|=\varrho}|\phi(\lambda) \psi(\lambda)| .
$$

It follows from the definition of $\psi$ and $\phi$ that for $|\lambda|=\varrho>1$,

$$
|\phi(\lambda) \psi(\lambda)|=\left|\lambda\left\langle(A-\lambda I)^{-1} u, u\right\rangle\right| \leq \varrho \frac{M}{\varrho-1} .
$$

Now it is easy to see that for $n>1, \varrho=1+1 / n, r=\varrho / 2$,

$$
\left|\left\langle A^{n} u, u\right\rangle\right| \leq \frac{2 a M}{n(\varrho-1)}\left(4 \varrho^{n}+\varrho^{n+1} \exp (8 a)\right) \leq 2 a e M\left(4+\left(\frac{n+1}{n}\right) e^{8 a}\right) .
$$

The obtained inequality holds obviously also for $n=1$. This ends the proof.

\section{References}

[1] I. Gohberg and M. G. Krein, Introduction to the Theory of Linear Nonselfadjoint Operators, Nauka, Moscow 1965 (in Russian).

[2] R. J. Leveque and L. N. Trefethen, On the resolvent condition in the Kreiss matrix theorem, BIT 24 (1984), 584-591.

[3] A. Pokrzywa, On continuity of spectra in norm ideals, Linear Algebra Appl. 69 (1985), $121-130$.

[4] M. N. Spijker, On the conjecture by LeVeque and Trefethen related to the Kreiss matrix theorem, report No. TW-90-07, Leiden Univ. Dept. of Math. and Comput. Sci. 